\title{
SENSITIVITY OF ULTRASOUND IN DIAGNOSIS OF BENIGN BILE DUCT PATHOLOGIES COMPARED TO MRCP
}

Prafulla KU. Dash', Sanket ${ }^{2}$

${ }_{1}^{1}$ Assistant Professor, Department of Radiodiagnosis, KIMS, Bhubaneshwar.

2Junior Resident, Department of Radiodiagnosis, SCBMCH, Cuttack.

\section{BACKGROUND}

ABSTRACT

Surgical or obstructive jaundice requires precise evaluation of the cause and level of obstruction to guide management. Ultrasound is routinely used as a first line imaging investigation in evaluation of biliary system, while MRCP is usually reserved for difficult or inconclusive cases.

\section{MATERIALS AND METHODS}

50 patients with clinical and biochemical features of obstructive jaundice were initially evaluated with Ultrasound. The patients with suspected benign pathologies were further investigated with MRCP for comparison using them later as gold standard reference.

\section{RESULTS}

Ultrasound has sensitivity of $81 \%$ in diagnosis of benign bile duct pathologies, highest sensitivity in detection of calculus disease and least in diagnosis of benign strictures.

\section{CONCLUSION}

Ultrasound has high sensitivity in detection and characterisation of benign bile duct pathologies. MRCP should be reserved for inconclusive cases, as routine addition does not seem to benefit in cases with diagnosis established with USG.

\section{KEYWORDS}

USG, MRCP, Choledocholithiasis, Obstructive Jaundice.

HOW TO CITE THIS ARTICLE: Dash PKU, Sanket. Sensitivity of ultrasound in diagnosis of benign bile duct pathologies compared to MRCP. J. Evolution Med. Dent. Sci. 2018;7(05):553-555, DOI: 10.14260/jemds/2018/125

\section{BACKGROUND}

Obstructive jaundice is a common surgical disorder and the management of which relies upon the preoperative diagnosis of not only the level of obstruction but also its cause, specifically to distinguish between malignant and benign pathologies.[1] Such patients are routinely evaluated by Ultrasound as the initial investigation. Magnetic resonance cholangiopancreatography (MRCP) is considered the gold standard investigation for the same. ${ }^{[2]}$ Both the studies share the advantage of being non-invasive, where Ultrasound has the distinct advantage of being more widely available and more affordable with more number of skilled sonographers that can interpret the findings.

\section{Aim}

To determine the sensitivity of USG in diagnosis of benign common bile duct pathologies, taking MRCP diagnosis as standard and to determine the percentage of patients that would be benefitted by using MRCP in addition to USG routinely.

'Financial or Other Competing Interest': None.

Submission 04-12-2017, Peer Review 27-12-2017,

Acceptance 30-12-2017, Published 29-01-2018.

Corresponding Author:

Sanket,

$P G$ in Department of Radiodiagnosis,

SCBMCH,

Cuttack-753007.

E-mail: dr.sanketkulkarni@gmail.com

DOI: $10.14260 /$ jemds $/ 2018 / 125$

(c) (i) (

\section{MATERIALS AND METHODS}

\section{Patients}

50 patients aged above 10 years were referred to Radiology for imaging evaluation of obstructive jaundice that fulfil the below criteria.

\section{Inclusion Criteria}

1. Chief complaint of Acute/ Chronic jaundice.

2. Laboratory workup showing obstructive aetiology with elevated levels of conjugated bilirubin.

\section{Exclusion Criteria}

1. Known/ suspected malignancy. Malignancy discovered on initial USG were excluded from the study.

2. Laboratory investigation showing Prehepatic/ Intrahepatic aetiology for jaundice.

3. Contraindications to MRI (Pacemaker, Orthopaedic implants, etc.)

4. Patients not consenting for the study.

Study Design- Descriptive Study.

Study Period- Jan. 2017 to July 2017.

Data Collection

All patients are subjected to-

1. Ultrasound evaluation with Philips HD7 machine with curvilinear 2-5 $\mathrm{MHz}$ probe with assistance of Tissue Harmonic Imaging (THI).

2. MRCP on GE 1.5T Machine. 
The findings of USG were compared to MRCP with MRCP findings being considered the gold standard.

\section{Statistical Method}

Sensitivity determination using confusion matrix.

\section{RESULTS}

Out of 50 people in the study 43 had choledocholithiasis (CBD stones), 4 had strictures, 1 had choledochal cyst and 2 had CBD masses not picked up on Ultrasound.

The distribution table and sensitivity values are shown in Table 1.

\begin{tabular}{|c|c|c|c|}
\hline & Ultrasound & MRCP & Sensitivity of USG \\
\hline CBD stone & 37 & 43 & $86 \%$ \\
\hline Stricture & 1 & 4 & $25 \%$ \\
\hline Other* & 1 & $3^{*}$ & $33 \%$ \\
\hline Total & $\mathbf{3 9}$ & $\mathbf{5 0}$ & $\mathbf{7 8 \%}$ \\
\hline Total Benign & 39 & 48 & $81.25 \%$ \\
\hline
\end{tabular}

Table 1. Distribution Table of Different Pathologies in the Current Study with Sensitivity of Ultrasound in Detecting the said Pathology

*Table includes 2 CBD masses that were not excluded based on our selection criteria and were incidentally picked up on MRCP.

\section{Stone Disease}

Out of 50 patients in our study, 43 patients showed CBD stones/ choledocholithiasis on MRCP. Out of the 43 patients, ultrasound could correctly diagnose 37 patients as having stone disease with a sensitivity of $86 \%$.

Out of the 6 cases of CBD calculi ultrasound showed calculi in gall bladder in 3 patients but failed to demonstrate CBD calculi. 3 of the 6 patients had no gall bladder calculi, but only isolated CBD calculi.

\section{CBD Stricture}

4 of 50 patients in our study had CBD strictures, out of which ultrasound could correctly identify only 1 case with a sensitivity of $25 \%$. All 4 of the patients were post cholecystectomy cases.

\section{Other Aetiologies}

One patient aged 16 years had Choledochal cyst, which was also picked up on ultrasound. Two other patients had CBD masses where ultrasound only showed biliary dilatation but no definitive mass, and hence were not excluded according to our exclusion criteria.

\section{Overall}

Out of the 50 cases, ultrasound could correctly diagnose 40 cases with an overall sensitivity of $78 \%$. As the total number of cases with benign aetiology on MRI were 48, sensitivity of ultrasound in diagnosing benign aetiologies was $81.25 \%$.

\section{Other Findings}

No false positive diagnosis was made on ultrasound.

\section{DISCUSSION}

Ultrasound is a widely available modality that can be used for evaluation of Common Bile Duct (CBD). USG study of CBD is performed with a curved array transducer $(2-5 \mathrm{MHz})$ in longitudinal and transverse planes with special attention to distal CBD near pancreatic head, which is best studied in transverse plane. Cut-off for dilation of CBD is $6 \mathrm{~mm}+1 \mathrm{~mm}$ every decade after the age of 6 years, and $10 \mathrm{~mm}$ post cholecystectomy. Stone disease on Ultrasound appears as an echogenic focus within the lumen casting posterior acoustic shadowing (PAS), but about $20 \%$ calculi may not cast PAS. Associated twinkle artefact of colour Doppler can assist in diagnosis. Benign strictures are difficult to recognise on ultrasound and generally appear as areas of abrupt tapering of the duct without any associated mass or calculus. Distal part of CBD is notoriously difficult to study on ultrasound due to bowel gas and obese body habitus. Using graded compression to displace bowel gas and Tissue Harmonic Imaging improves visualisation of CBD.

MRCP is considered the gold standard for diagnosis of CBD pathology with sensitivity on par with ERCP (Endoscopic retrograde cholangiopancreatography). Imaging in MRCP is done using highly T2-weighted sequences. Non-invasive nature, lack of radiation and lesser complication makes it more preferable to ERCP. Commonly used are 3D SSFP (Steady State Free Precession) sequence and FSE (Fast Spin Echo) sequences like HASTE (Half-Fourier Acquisition SingleShot Turbo Spin Echo) and RARE (Rapid Acquisition with Rapid Enhancement sequence). On MRCP, Choledocholithiasis appears as filling defects in high intensity fluid of CBD. Benign strictures appear as abrupt cut-off of the duct. However, MRCP has its inherent artefacts like motion artefacts, susceptibility artefacts, flow artefacts and potential pitfalls like vascular impressions and pseudocalculus sign of sphincter contraction.

Ultrasound is generally regarded as first line investigation in evaluation of the biliary system. Advent of better ultrasound technology, especially Tissue Harmonic Imaging (THI) has resulted in improved visualisation of the biliary system.[3] However, studies have cited a wide range of sensitivity from as low as $20 \%$ to $80 \%$. Older studies by Todua et al have shown sensitivity of USG to range between $23 \%-85 \% .{ }^{[4]}$ Another relatively recent study by Singh A et al, a sensitivity of $93 \%$ was reported in diagnosis of choledocholithiasis.[5] Our study result also shows a high sensitivity of $86 \%$ in the diagnosis of choledocholithiasis consistent with recent studies.

Al-Obaidi et al reported a sensitivity of $44 \%$ for USG diagnosis of stricture.[6] Our study result showed only 25\% sensitivity, possibly due to smaller sample size.

In those cases where the cause of diagnosis was established definitively on ultrasound, MRCP only confirmed the diagnosis without contradicting ultrasound diagnosis.

\section{CONCLUSION}

In patients with biliary obstruction with a benign pathology, Ultrasound is a good initial imaging modality with high sensitivity. However, due to operator dependence and inherent limitations of the technology, variable detection rates are seen. In small number of patents with inconclusive ultrasound, MRCP can help definitively establish the aetiology. The patients in which ultrasound established the aetiology, addition of MRCP routinely does not seem to be beneficial. 


\section{REFERENCES}

[1] Wang L, Yu W. Obstructive jaundice and perioperative management. Acta Anaesthesiol Taiwan 2014;52(1):22-9.

[2] Raju AP, Divyashree, Mehrotra. Role of MRI-MRCP in evaluation of obstructive Jaundice. J Med Sci Clin Res 2017;05(03):18310-5.

[3] Choudhry S, Gorman B, Charboneau JW, et al. Comparison of tissue harmonic imaging with conventional US in abdominal disease. Radiographics 2000;20(4):1127-35.
[4] Todua FI, Karmazanovskii GG, Vikhorev AV. Computerized tomography of the mechanical jaundice in the involvement of the distal region of the common bile duct. Vestn Roentgenol Radiol 1991;2:15-22.

[5] Singh A, Mann HS, Thukral CL, et al. Diagnostic accuracy of MRCP as compared to ultrasound/CT in patients with obstructive Jaundice. J Clin Diagn Res 2014;8(3):103-7.

[6] Al-Obaidi S, Al-Hilli MRA, Fadhel AA. The role of ultrasound and magnetic resonance imaging in the diagnosis of obstructive Jaundice. The Iraqi Postgraduate Medical Journal 2007;6(1):7-17. 